\title{
THE EQUIVALENCE PROBLEM FOR DETERMINISTIC TOL-SYSTEMS IS UNDECIDABLE
}

\author{
Grzegorg ROZENBERG \\ Department of Con puter Science. State University of New York at Buffalo, \\ $\$ 226$ Ridgr l.ea Road, Amherst. New York 14226. U.S.A. \\ and \\ Mathematical Institutc, Utrecht State University. Utrecht -. Uithof, Netherlands
}

Recerved 17 April 1972

\section{Introdurtion}

$L$-languages were introduced by Lindermayer [3] for the description of the development of filamentous organisms. Originally they were described in terms of linear arrays of automata, but in later works the formaliam was changed to the more linguistical notion of an L-system. This gave rise to various families of developmental languages with an already fairiy developed literature (see, e.g., ref. [4]).

This paper continues resear, ti on TOL-systems and languages introduced in ref. [6].

A TOL-system has the following components:

(i) A finite set of symbols, $\Sigma$ the alphabet;

(ii) A starting string, $\omega$, the axiom;

(iii) A finite collection P of tables, each of which is a finite set of productions which tell us by what strings in $\Sigma^{*}$ a yymbol may be replaced. A table may, in general, contain several productions allowing to replace the same symbol. In every step of a derivation, all symbols in a string must be simultanecuslv laced according to the production rules, 11 of which are chosen from one (but arbitrary) table.

The language generated by the given TOL-system consists $c$. ail strings which can be derived from $\omega$ in a finite number of steps.

One of the important problems, from both, the biological and formal lan $n_{b}$ uage theory $f$-ints of view, is the equivalence problem for TOL-systems, which can be phrased as follows: Does there exist an algorithn, which, given arbitrary TOL-systems $G_{1}$ and $G_{2}$, will decide in a tinite number of steps whether or not the languages generated by $G_{1}$ and $G_{2}$ are identical?

ln this note we prove that such an algorithm cannot exist even for deterministic TOL-systems, i.e., such TOL-systems, where each table provides exactly one possibility of rewriting for every letter in the alphabet of the given system.

Recently such a result was obtained hy Meera Blattner [7] for OL-systems, i.e., such TOL-systems which contain only one table. The result presented here is however independent in the following sense:

(i) It was proved in ref. [6] (1972), that the classes of $\mathrm{OL}$ and deterministic TOL languages are incomparable but not disjoint.

(ii) We prove our result by a direct construction (based on a simulation of the post correspondence problem).

\section{Definitions}

For the sake of completeness. we shall $\pi_{\mathrm{r}}$ eat (afier ref. [6] ) definitions of TOL-systems, languages. etc., as well as the post corresponden,e theorem (after ref. [1]). Our notation for basic te.ms from a for- 
mal language theory will follow this of ref. [1].

\section{Definition 1}

A TOL-system (over an alphabet $\Sigma$ ) is defined as a construct $G=\langle\Sigma, P, \sigma\rangle$, where

1. $\Sigma$ is a finite set (called the alphabet of $G$ ).

2. $P$ is a finite set, $P=\left\{T_{1}, \ldots, T_{f}\right\}$ for some $f \geqslant 1$, each element of which is a finite subset of $\Sigma \times \Sigma^{*}$.

Psatisfies the following (completeness) condition:

$$
(\forall T) p(\forall a)_{\Sigma}(\exists \alpha)_{\Sigma^{*}}(\langle a, \alpha\rangle \in T) .
$$

$P$ is callec the set of tables of $G$.

$\because \sigma \in \Sigma^{+}$(called the axiom of $G$ ).

We assume that both $\Sigma$ and $P$ are non-empty sets.

\section{Definition 2}

Let $G=(\Sigma, P, \sigma)$ be a T0L-system. Le $i$

$$
x \in \Sigma^{+}, x=a_{1} \ldots a_{k},
$$

where each $a_{j}, 1 \leqslant j \leqslant k$, is an element of $\Sigma$, and let $y \in \Sigma^{*}$. We say that $x$ directly derives $y$ in $G\left(x \Rightarrow_{G} y\right)$ if, and only if, there exist $T$ in $P$ and $p_{1}, \ldots, p_{k}$ in $T$ such that

$$
p_{1}=\left\langle a_{1}, \alpha_{1}\right\rangle, p_{2}=\left\langle a_{2}, \alpha_{2}\right\rangle, \ldots, p_{k}=\left\langle a_{k}, \alpha_{k}\right\rangle
$$

(for some $\alpha_{1}, \ldots, \alpha_{k} \in \Sigma^{*}$ ) and $y=\alpha_{1} \ldots \alpha_{k}$. We say that $x$ derives $y$ in $G\left(x \Rightarrow_{G} * y\right)$ if, and only if, either (i) there exists a sequence of words $x_{0}, x_{1}, \ldots, x_{n}$ in

$$
\Sigma^{*}(n \geqslant i) \text { such that } x_{0}=x, x_{n}=y \text { and } x_{0}
$$

(ii) $x=y$.

$$
{ }_{G} x_{1}{ }_{G} \cdots{ }_{G} x_{n} \text {, or }
$$

${ }_{G}$ simply denotes the transitive and reflexive clowire of the relation $G^{\cdot} \Rightarrow G^{+}$shall denote the transitive cloeu: of $\Rightarrow G$.

\section{Definition 3}

Let $G=\langle\Sigma, P, \sigma\rangle$ be a TOL-system. The language of $G$ (denoted as $L(G)$ ) is defined as

$$
L(G)=\left\{x: 0 \Rightarrow G^{* x}\right\},
$$

\section{Irfinition 4}

Let $\Sigma$ be a finite alphabet and $L \subseteq \Sigma^{*} . L$ is called a TCL language if, and only if, there exists a TOL. grammar $G$ such that $L(G)=L$.

\section{Definition 5}

A TOL-system $G=\langle\Sigma, P, \sigma\rangle$ is called deterministi(denoted as DTOL) if, and only if, for each $T$ in $P$ and each $a$ in $\Sigma$ there exists exactly one element $\langle A, \alpha\rangle$ in $T$ such that $A=a$.

\section{Notation}

Let $G=(\Sigma, P, a)$ be a TOL syster, If $(a, a)$ is an element of some $T$ in $P$, then we call it a production (for $a$ in $T$ ) and write $a \rightarrow \alpha \in T$, or $a \rightarrow T \alpha$. If $x \Rightarrow G y$ "using" a table $T$ of $P$, then sometimes we shall write $x \Rightarrow T Y$.

\section{Theorem I (Post correspondence theorem)}

Let $\Sigma$ contain at least two elements. Then it is recursively unsolvable to determine for arbitrary $n$. tuples $\left(\alpha_{1}, \ldots, \alpha_{n}\right)$ and $\left(\beta_{1}, \ldots, \beta_{n}\right)$ of nonemply words over $\Sigma$, whether there exists a (nonempty) sequence of indices $i_{1}, \ldots, i_{k}$ such that $\alpha_{i} \cdots \alpha_{i k}=\beta_{i} \cdots \beta_{i k}$.

The reader is referred to ref. [5] or [2] for the proof of this theorem.

\section{Result}

\section{Theorem 2}

It is recursively undecidable to dec (Mnine for arbitrary DTOL-systems $G_{1}, G_{2}$ phathe $g\left(G_{1}\right)=L\left(G_{2}\right)$. Proof (outline):

Let $\Sigma$ be a finite alphabet such that $¥ \Sigma>2$, say $\Sigma=\left(a_{1}, \ldots, a_{n}\right\}$ for some $n \geqslant 2$. Let $L_{1}, L_{2}$ be an instance of the post correspundence problem over an alphabet $\Sigma$, say $L_{1}=\left(\alpha_{1}, \ldots, \alpha_{m}\right), L_{2}=\left(\beta_{1}, \ldots, \beta_{m}\right)$ for some $m>2$. Let $G_{1}=\left\langle V, \mathbb{P}_{1}, \omega\right\rangle$ be a DTOL sys. tem where

I. $V=\Sigma \cup\left\{S_{1}, S_{2}, R_{1}, R_{2}, U_{1}, U_{2}, A_{1}, A_{2}\right\} \cup\{1$, ..., $m\}$, where we assume that

$$
\Sigma \cap\left(\left\{S_{1}, \ldots, A_{2}\right\} \cup\{1, \ldots, m\}\right)=
$$

II. $\omega=S_{1} S_{2}$.

III. $P_{1}$ consists of the foll owitig tables (which, for convenionce are listed in groups):

0) $T_{0}=\left\{S_{1} \rightarrow A_{1}, S_{2} \rightarrow A_{2}, X \rightarrow X\right.$ for every $X$ in $\left.V-\left\{s_{1}, s_{2}\right\}\right\}$.

1) For every $1 \leqslant i \leqslant m, 1 \leqslant k, 1 \leqslant n$, such that $k \neq l$, let 
$T_{1, i, k . l}=\left\{S_{1} \rightarrow i R_{1} a_{k}, S_{2} \rightarrow i R_{2} a_{1}, X \rightarrow X\right.$

for every $X$ in $\left.V-\left(S_{1}, x_{2}\right)\right\}$

2) For every $1 \leqslant i \leqslant m, 1 \leqslant i \leqslant n$, let

$$
T_{2, i, j}=\left(s_{1} \rightarrow i U_{1} a_{j} . s_{2} \rightarrow i U_{2} a_{j}, x \rightarrow x\right.
$$

for every $X$ in $\left.V-\left\{s_{1} . s_{2}\right\}\right\}$.

3) For every $1 \leqslant i \leqslant m .1 \leqslant i \leqslant n$, let

$T_{3, j, 1}=\left\{U_{1} \rightarrow U_{1} a_{j}, U_{2} \rightarrow i U_{2} a_{j}, X \rightarrow X\right.$

for every $X$ in $V-\left\langle U_{1}, U_{2}\right)$.

$T_{3,0\}}=\left\{U_{1} \rightarrow U_{1} a_{i}, U_{2} \rightarrow U_{2} a_{j}, X \rightarrow X\right.$

fot every $X$ in $V-\left\{U_{1}, U_{2}\right)$

4) Sor every $1 \leqslant i \leqslant m, 1<k, 1 \leqslant n$, such that $k * t$, lel

$T_{4, a, k, 1}=\left(U_{1}-R_{1} a_{k}, U_{2} \rightarrow R_{2} a_{1}, X \rightarrow X\right.$

for every $X$ in $\left.V-\left\{U_{1}, U_{2}\right\}\right)$.

$$
T_{\left.4, i_{1}, k_{1}\right)}=\left\{U_{1} \rightarrow i R_{1} a_{k}, U_{2} \rightarrow i R_{2} a_{1}, X \rightarrow X\right.
$$

for every $X$ in $V-\left\{U_{1}, U_{2}\right\}$.

5) For every $i, i, k,{ }^{\prime}$ such that $i, j \in\{0, \ldots, m\}$, $k, l \in(0, \ldots, n), l \in t$

$$
T_{S, i_{1} k_{1},}=\left(R_{1} \rightarrow I_{i} R_{1} v_{k}, R_{2} \rightarrow I_{i} R_{2} v_{l}, X \rightarrow X\right.
$$

for every $X$ in $\left.V-\left\{R_{1}, R_{2}\right\}\right\}$,

where $t_{0}=v_{0}=\Lambda, t_{i}=i$ for $1 \leqslant i \leqslant m, v_{i}=a_{i}$ for $1<j<n$.

6) $T_{6}=\left\{R_{1} \rightarrow A_{1}, R_{2} \rightarrow A_{2}, X \rightarrow X\right.$ for every $X$ in $\left.V-\left\{R_{1}, R_{2}\right\}\right\}$.

The following result is a direct consequence from the construction of $\boldsymbol{G}_{1}$, and its (rather long but straight. forward) iormal proof is left to the reader.

\section{Lemme :}

$$
L\left(C_{1}\right) \cap\left(\Sigma \cup\{1, \ldots, n\} \cup\left\{A_{1}, A_{2}\right\}\right)^{*}=
$$

$=A_{1} A_{2} \cup\left(x A_{1} y x A_{2} \bar{v} x \in\{1, \ldots, m\}^{+}, y, \bar{y} \in \Sigma^{+}\right.$, $y \neq \vec{y}$. Let for ever $\quad<i \leqslant m$.

$$
T_{7, i}=\left\{A_{1} \rightarrow i A_{1} \alpha_{i}, A_{2} \rightarrow i A_{2} \beta_{i}, X \rightarrow X\right.
$$

for every $X$ in $V-\left\{A_{1}, A_{2}\right\}$.

Let $G_{2}=\left\langle\dot{\Delta}, P_{2}, S_{1} S_{2}\right\rangle$ be a DTOL-system such that

$$
P_{2}=P_{1} \cup\left\{T_{7, i}: 1 \leqslant i \leqslant m\right\}
$$

\section{Lemmo 2}

$L\left(G_{1}\right)=L\left(G_{2}\right)$ if, and only if, there does not exis: a solution for the $L_{1}, L_{2}$ instance of the post correspondence problem.

Proof:

(i) Let us assume that $L\left(C_{1}\right)=L\left(G_{2}\right)$. Then from lemma 1 , it follows that for every word (in $L\left(G_{1}\right)$ ) of the form $x A_{1} y x A_{2} \bar{y}$, where $x \in\{1, \ldots, m\}, y$, $\bar{y} \in \Sigma^{+}$we have $y \neq \frac{2}{y}$. But, from the construction of tables $T_{7, i}(1 \leqslant i \leqslant m)$, it follows that for every sequence $i_{i}, \ldots, i_{f},(f \geq 1)$, of elements from $\{1, \ldots, m\}$,

$$
i_{1} \cdots i_{f} A_{1} \alpha_{i_{f}} \cdots \alpha_{i_{1}} i_{1} \cdots i_{f} A \beta_{f} \cdots \beta_{1}
$$

is in $L\left(G_{2}\right)$. Hence for every sequence $i_{1}, \ldots, i_{f}$, $(f>1)$, of elements from $\{1, \ldots, m\}, \alpha_{i f} \cdots \alpha_{i,} \neq$ $\neq \beta_{i} \cdots \beta_{i_{1}}$, and so the post correspondence problem has no solution for the instance $L_{1}, L_{2}$.

(ii) Let us assume that the post correspondence problem has no solution for the instance $L_{1}, L_{2}$, which means that for every sequence $i_{1}, \ldots, i_{f}$, $(f \geq 1)$, of elements from $\{1, \ldots, m\}, \alpha_{i_{1}} \cdots \alpha_{i_{f}} \neq$ $\neq \beta_{i} \cdots \beta_{i f}$.

But, from the construction of $T_{7, i}($ for $1 \leqslant i \leqslant m)$ it follows that

$$
\begin{aligned}
L\left(G_{2}\right)-L\left(G_{1}\right) & =\left\{i_{1} \cdots i_{p} A_{1} \alpha_{i_{p}} \cdots \alpha_{i_{1}} i_{1} \cdots i_{p} A_{2} \beta_{i_{p}} \cdots \beta_{i_{1}}:\right. \\
\text { where } p & \left.\geqslant 1, i_{1} \ldots, i_{p} \in\{1, \ldots, m\}\right\},
\end{aligned}
$$

and so (see lemma 1), $L\left(G_{2}\right)-L\left(G_{1}\right) \subseteq L\left(G_{1}\right)$, hence $L\left(G_{1}\right)=L\left(G_{2}\right)$.

From (i) and (ii) lemma 2 follows.

From lemma 2, we see, that, if the equivalence problem for DTOL-systems is recursively solvable, then the post correspondence problem is also recurs- 
Wely solvable, this is a contradiction. Thus, theorem itholds.

\section{Corollory}

It is recursive:y unsolvable to determine for arbitraty ToL-systenis $G_{1}, G_{2}$ whether $L\left(G_{1}\right)=L\left(G_{2}\right)$. Proof:

This follows from definitions of TOL and DTOL. systems (egeh DTOL-system is also a TOL-system).

\section{Solnowledlgement}

The author is indebted to the re: iree for useful comments. The research for this paper has been supperted by NSF Grant GJ998.

\section{References}

[1] S. Ginsburg. The riathematical theory of context free languages (McGraw-Hill, New York, 1966).

[2] J.E. Hopcro:t, J.D. Ullman. Formal languages and their relation to automata (Addison-Wesley, Reading, Massachusetts, 1969).

(3) A. Lindenmayer, Mathematical models for cellular interactions in development, Parts I and II, J. Theoret. Biol., 18 (1968) 280.

[4] A. Lindenmayer, G. Rozenbers, Developmental systems and languages, in: Proc. ACM Symposium on Theory of Computing (1972).

[5] E.L. W.st, A variant of a recurgivaly unsolvable problem, Bull. Am. Math. Soc. 52 (1946) 264.

[6] G. Rozenberg, TOL systems and languages, Information and Control to appear (1972).

[7] S. Greibach, private communication. 